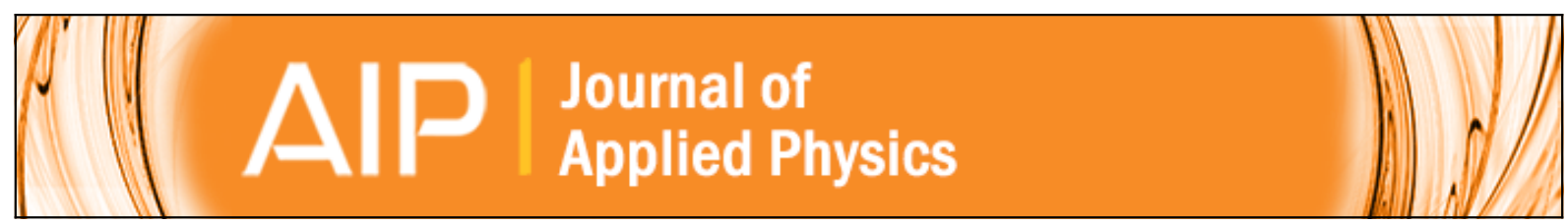

\title{
Three dimensional simulation of giant magneto-impedance effect in thin film structures
}

Bodong Li and Jürgen Kosel

Citation: Journal of Applied Physics 109, $07 \mathrm{E} 519$ (2011); doi: 10.1063/1.3556947

View online: http://dx.doi.org/10.1063/1.3556947

View Table of Contents: http://scitation.aip.org/content/aip/journal/jap/109/7?ver=pdfcov

Published by the AIP Publishing

\section{Articles you may be interested in}

Magnetoimpedance effect at the high frequency range for the thin film geometry: Numerical calculation and experiment

J. Appl. Phys. 116, 243904 (2014); 10.1063/1.4904960

Magnetoelectric coupling of multiferroic chromium doped barium titanate thin film probed by magneto-impedance spectroscopy

Appl. Phys. Lett. 104, 142901 (2014); 10.1063/1.4870263

Enhanced magnetoelectric effect in three-phase Mn Zn Fe 2 O 4/Tb $1-x$ Dy x Fe 2 - y/Pb ( Zr , Ti ) O 3 composites

J. Appl. Phys. 100, 124108 (2006); 10.1063/1.2402968

Giant magnetoimpedance in trilayer structures of patterned magnetic amorphous ribbons

Appl. Phys. Lett. 81, 1654 (2002); 10.1063/1.1499769

Magneto-impedance effects in multilayered permalloy materials

J. Appl. Phys. 86, 1057 (1999); 10.1063/1.370847

MIT LINCOLN

LABORATORY CAREERS

Discover the satisfaction of innovation and service

to the nation
- Space Control

- Air \& Missile Defense

- Communications Systems \& Cyber Security

- Intelligence, Surveillance and

Reconnaissance Systems

- Advanced
Electronics
- Tactical Systems
- Homeland
Protection
- Air Traffic Control

LINCOLN LABORATORY

MassachusetTs Institute of TeChNOLOgY

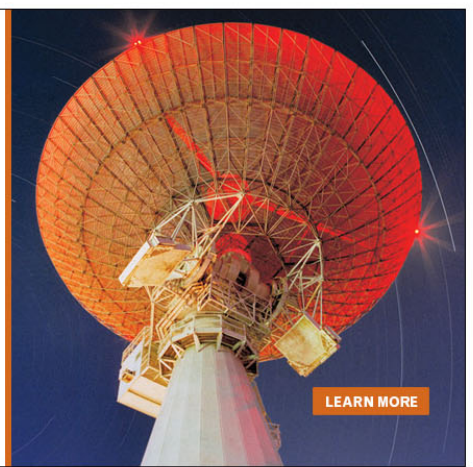




\title{
Three dimensional simulation of giant magneto-impedance effect in thin film structures
}

\author{
Bodong $\mathrm{Li}^{\mathrm{a})}$ and Jürgen Kosel \\ Physical Sciences and Engineering Division, King Abdullah University of Science and Technology, Thuwal \\ 23955-6900, Kingdom of Saudi Arabia
}

(Presented 17 November 2010; received 24 September 2010; accepted 30 November 2010; published online 4 April 2011)

\begin{abstract}
In this paper, a three dimensional model for the giant magneto-impedance (GMI) effect in thin film structures is developed using the finite element method (FEM) with a GMI permeability model embedded. One-layer, three-layer, and five-layer thin film structures are simulated. The GMI effect and the sensitivity are calculated as a function of the external magnetic field, driving frequency, and the thickness of the magnetic layers. The results show that the five-layer structure has the best performance, which is in accordance with experimental results. The GMI ratio and the sensitivity first improve with the increasing thickness of the magnetic layer but reach saturation at a certain value of the thickness. In a five-layer structure, saturation of the GMI effect becomes effective at about $3 \mu \mathrm{m}$ thickness of the magnetic layers, where a GMI ratio of $1125 \%$ was obtained, with a corresponding sensitivity of $0.37 \% / \mathrm{A} / \mathrm{m}$ (29.6\%/Oe). (C) 2011 American Institute of Physics. [doi:10.1063/1.3556947]
\end{abstract}

\section{INTRODUCTION}

As a potential technology for next generation, magnetic microsensors with a high sensitivity to low magnetic field changes, the giant magneto-impedance (GMI) effect has attracted increasing interest in the past decade. The GMI effect was originally found in ferromagnetic microwires, which, so far, proved to have the best performance compared to other GMI structures. However, the fabrication technology of the microwires adds complexity and costs to the production of an integrated sensor. Consequently, more and more effort has been put into the research of thin film GMI structures in order to improve the sensitivity.

A single layer thin film GMI structure consists of a soft magnetic thin film with magnetic domains transversal to the longitudinal axis. ${ }^{1}$ An alternating current $I_{\text {ac }}$ generates a transverse flux $B_{\text {tran }}$ inside and around the film. After applying an external field $H_{\text {ext }}$, domain wall motion as well as magnetization rotation contribute to a permeability change in the transversal direction, leading to a change in impedance. Based on the single layer GMI structure, several other configurations such as three- and five-layer structures have been developed to improve the GMI performance. ${ }^{2-4}$ In these structures, improvement is achieved by reducing the base impedance using a highly conductive metallic inner layer and isolating the conductive layer from the magnetic ones. ${ }^{5,6}$

In general, the dependence of the permeability on $H_{\text {ext }}$ can be obtained by solving the Landau-Lifshitz equation. Much work has been done previously to develop permeability models and simulate the impedance change in different GMI structures. ${ }^{7-10}$ However, analytical solutions of the GMI effect for more complex structures are difficult to obtain. In this case, the finite element method (FEM) is a

\footnotetext{
a) Author to whom correspondence should be addressed. Electronic mail: bodong.li@kaust.edu.sa.
}

suitable method to evaluate the impedance change of the GMI structure, providing flexibility with respect to the design of the structures. Previous work, using FEM, focused on the skin effect in a two dimensional (2D) model $^{11}$ without considering the dependence of the permeability on the external field.

In this paper, the FEM is applied in combination with previously developed permeability models ${ }^{9}$ for the GMI effect in order to study the performance of GMI thin film structures. The 3D models for one-, three-, and five-layer structures are developed, and the dependencies of the GMI effect and the sensitivity on the geometrical parameters as well as the frequency are calculated.

\section{METHOD}

The GMI ratio is the relative change of the impedance with respect to the external magnet field

$$
\frac{\Delta Z}{Z}(\%)=100 \% \times \frac{Z\left(H_{\mathrm{ext}}\right)-Z\left(H_{0}\right)}{Z\left(H_{0}\right)},
$$

where $Z\left(H_{\text {ext }}\right)$ is the impedance under a certain external field $H_{\text {ext }}$ and $H_{0}$ is the zero external magnetic field.

The sensitivity is defined by

$$
\zeta=(100 \% \times) \frac{Z\left(H_{2}\right)-Z\left(H_{1}\right)}{Z\left(H_{1}\right)} /\left(H_{2}-H_{1}\right) .
$$

The impedance in the ferromagnetic thin film can be expressed as

$$
Z=\frac{V_{\mathrm{ac}}}{I_{\mathrm{ac}}\left(H_{\mathrm{ext}}\right)},
$$

where $V_{\mathrm{ac}}$ is the voltage applied to the sensor and $I_{\mathrm{ac}}$ is a function of $H_{\text {ext }}$. 


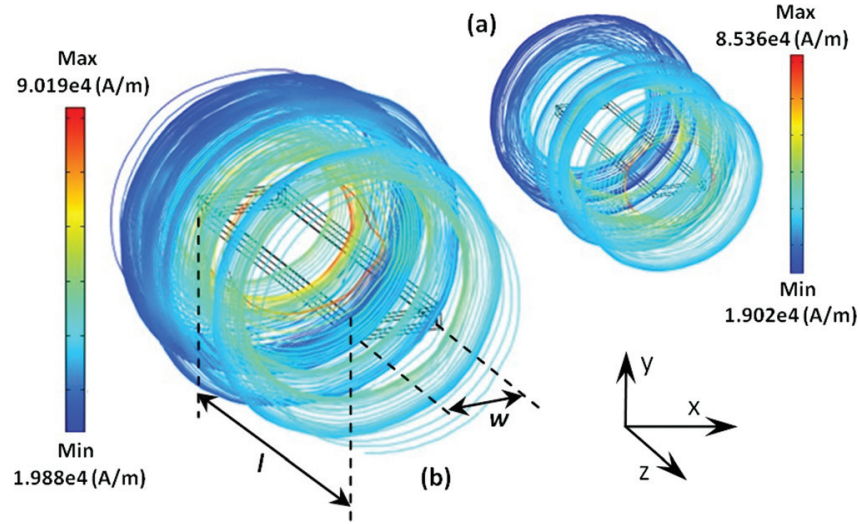

FIG. 1. (Color online) A 3D plot of the circumferential magnetic flux of the (a) three-layer and (b) five-layer thin film GMI sensors.

The 3D finite element analysis was carried out using a commercial software (COMSOL-multiphysics). ${ }^{12}$ A value of $V_{\text {ac }}=5 \mathrm{~V}$ was defined as a boundary condition on one end of the sensor, whereas the other end was set to the ground potential. In the case of five-layer structure, $V_{\mathrm{ac}}$ was applied only to the metal layer. In order to find $I_{\mathrm{ac}}$, the permeability dependence of the ferromagnetic material on the external field is required. We applied the permeability model developed previously. ${ }^{9}$

$$
\mu=\frac{\gamma \cdot M_{s}\left(\gamma \cdot H_{\mathrm{equ}}+i \alpha \omega\right) \sin ^{2}\left(\theta+\theta_{0}\right)}{\left(\gamma \cdot H_{\mathrm{equ}}+i \alpha \omega\right)^{2}-\omega^{2}},
$$

where $\omega$ is the angular frequency, $\theta$ is the angle between $M_{s}$ and the easy axis, $\theta_{0}$ is the small angle existing between the direction of the ideal anisotropy and the real easy axis, and $H_{\text {equ }}$ is the magnetic field component that affects the saturation magnetization. Under the assumption of a magnetic anisotropy in transversal direction, the third order permeability tensor for the model reduces to

$$
\left[\begin{array}{lll}
\mu & 0 & 0 \\
0 & \mu & 0 \\
0 & 0 & 1
\end{array}\right] .
$$

The three different thin film structures-one-, three-layer, and five-layer-that were investigated showed dimensions of width $w=50 \mu \mathrm{m}$ and length $l=200 \mu \mathrm{m}$ (Fig. 1). A thickness of $t_{\mathrm{mag}}=1 \mu \mathrm{m}$ and $t_{\mathrm{met}}=4 \mu \mathrm{m}$ was assigned to the magnetic layer and the conducting layer, respectively. However, the

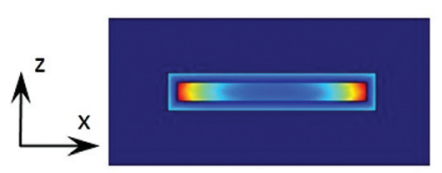

(a)

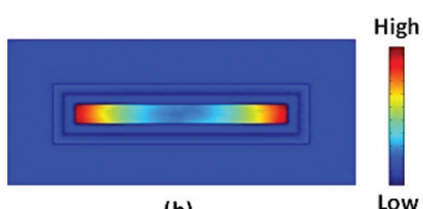

(b)

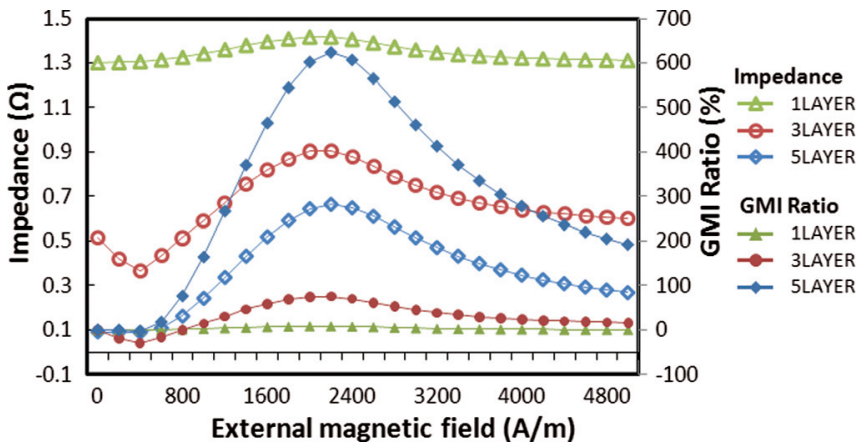

FIG. 3. (Color online) Impedance change and GMI ratio as a function of the external magnetic field for one-, three-, and five-layer thin film structures.

influence of different values of $t_{\mathrm{mag}}$ was also investigated. The isolation layer was set as $\mathrm{SiO}_{2}$. The conductivities of the ferromagnetic and conducting layer were $7.69 \times 10^{5} \mathrm{~S} / \mathrm{m}$ $\left[(\mathrm{CoFe})_{80} \mathrm{~B}_{20}\right]$ and $4.56 \times 10^{7} \mathrm{~S} / \mathrm{m}(\mathrm{Au})$, respectively. All the parameters including $M_{s}=5.6 \times 10^{5} \mathrm{~A} / \mathrm{m}, \quad \gamma=2.2 \times 10^{5}$ $\mathrm{m} / \mathrm{As}, \alpha=0.3$, and $\theta_{0}=\pi / 180$ were taken from literature. ${ }^{10}$

The simulations were carried out for values of $H_{\text {ext }}$ from 0 to $4800 \mathrm{~A} / \mathrm{m}$ and, if not stated otherwise, with a driving frequency of $80 \mathrm{MHz}$.

\section{RESULTS}

The circumferential magnetic flux of the three- and fivelayer GMI thin film structures are presented in Fig. 1. The circumferential magnetic flux generated by the five-layer structure is stronger than the three-layer structure, which is a result of the higher current density obtained in the five-layer structure. Figure 2 shows the current density distribution along the cross sections of the three-layer and five-layer structures. As can be seen, the insulation layer in the fivelayer structure prevents the current from leaking into the magnetic layer. This current leakage causes an increase of the impedance of the three-layer structure compared to the five-layer structure.

The impedance and the corresponding GMI ratios as a function of $H_{\mathrm{ext}}$ are presented in Fig. 3. The results show that the single layer GMI has the highest absolute impedance but the lowest GMI ratio. The GMI ratio of the single layer structure is in good agreement with the results found previously, ${ }^{10}$ which is less than $10 \%$ at $80 \mathrm{MHz}$ for this specific material.

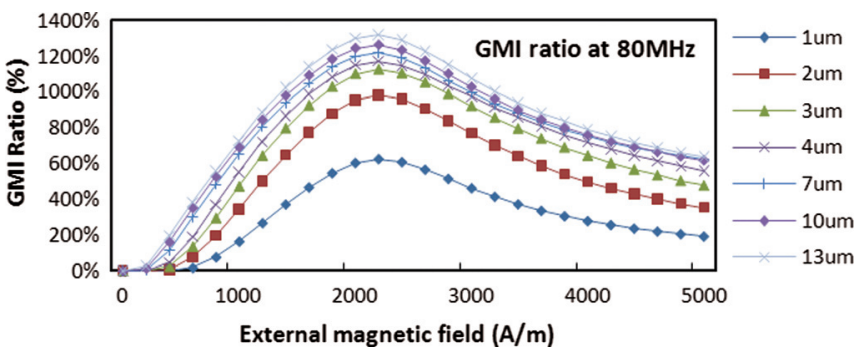

FIG. 4. (Color online) Dependence the GMI ratio of the five-layer structure with different values of the thickness of the magnetic layer as a function of the external field.
FIG. 2. (Color online) Current density distribution along the cross section under the external field of $2200 \mathrm{~A} / \mathrm{m}$ in a (a) 3-layer and (b) 5-layer GMI structure. 


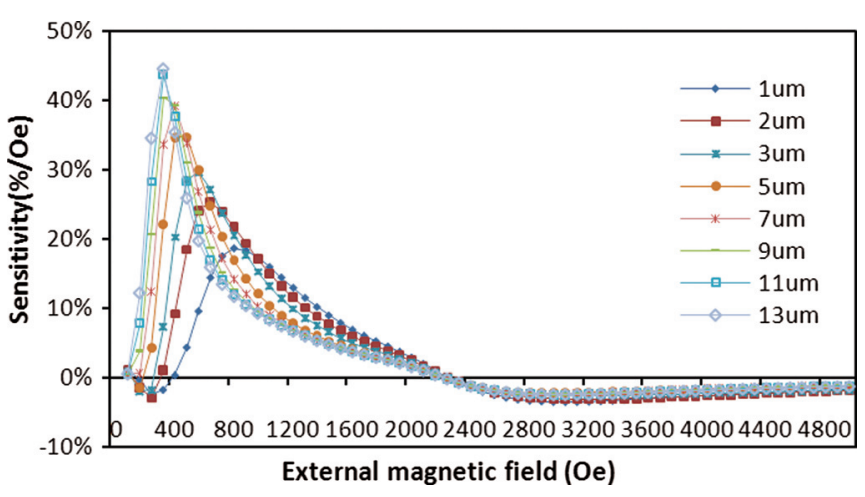

FIG. 5. (Color online) Sensitivity of the five-layer GMI structure with different values of the thickness of the magnetic layer and as a function of the external field.

The five-layer structure is superior to the other two structures and results in the highest GMI ratio of up to $625 \%$.

Figure 4 shows the influence of $t_{\text {mag }}$ on the GMI ratio for a five-layer structure. An increased thickness yields an increase of the GMI ratio, and the maximum GMI ratio found was $1321 \%$ at $H_{\text {ext }}=2200 \mathrm{~A} / \mathrm{m}$. As for the sensitivity (Fig. 5), an increased thickness also resulted in an increased value. The sensitivity reaches $0.55 \% / \mathrm{A} / \mathrm{m}(44 \% / \mathrm{Oe})$ at the thickness of $13 \mu \mathrm{m}$. Interestingly, a maximum sensitivity is obtained at lower values of the field as the thickness increases. It is also observed that the sensitivity shows negative values at weak magnetic fields. This behavior is a result of the permeability model, in which the real part of the permeability shows a decrease for small values of the magnetic field. Figure 6 shows the maximum values of the GMI ratio and the sensitivity as a function of $t_{\text {mag }}$. It is evident that the increase of both the GMI ratio and the sensitivity with $t_{\text {mag }}$ approaches saturation. This is an important finding considering the challenges associated with the fabrication of thin films with a thickness of several micrometers. From this result, a thickness of $t_{\text {mag }}=3 \mu \mathrm{m}$ seems to be most effective because a further increase in thickness causes only a small increase of the GMI ratio. The sensitivity shows a slower

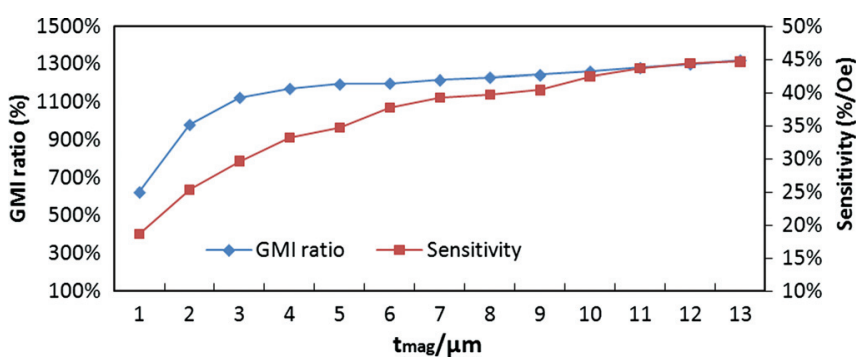

FIG. 6. (Color online) Maximum GMI ratio and sensitivity of the five-layer GMI structure as a function of the thickness of the magnetic layer.

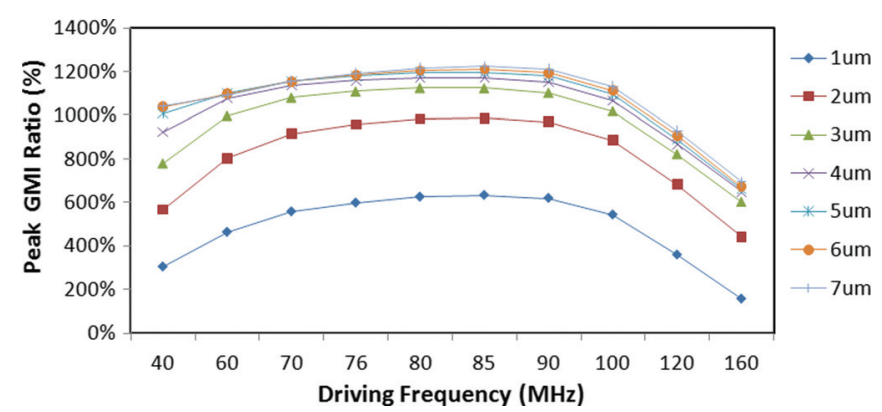

FIG. 7. (Color online) Dependence of peak GMI ratio of the five-layer GMI structure with different values of the thickness of the magnetic layer as a function of the driving frequency.

approach toward saturation, and a thickness beyond $t_{\text {mag }}=3 \mu \mathrm{m}$ might still be beneficial. In Fig. 7 , the GMI dependence on the driving frequency and $t_{\text {mag }}$ is shown. The results show that the optimal frequency range of this fivelayer structure is almost independent of the thickness of the magnetic layer and lies between 80 and $90 \mathrm{MHz}$.

\section{CONCLUSION}

A 3D model has been developed using the finite element method to determine the GMI ratio and the sensitivity in different thin film GMI structures. The current density distribution and the skin effect were observed and discussed. The results confirmed that the five-layer structure has superior performance, which is in accordance with experimental results, and the optimum frequency with respect to the GMI ratio is between 80 and $90 \mathrm{MHz}$. A study on the influence of the thickness of the magnetic layer on the GMI ratio as well as the sensitivity was carried out. Both were found to first increase with the increasing thickness and then approach saturation. This finding provides valuable information for efficient fabrication of GMI sensors.

${ }^{1}$ L.V. Panina, K. Mohri, and T. Uchiyama, Phys. A 241, 429 (1997).

${ }^{2}$ L.V. Panina and K. Mohri, Appl. Phys. Lett. 65, 1189 (1994).

${ }^{3}$ N. A. Usov, A. S. Antonov, and A. N. Lagar'kov, J. Magn. Magn. Mater. 185, 159 (1998)

${ }^{4}$ T. Morikawa, Y. Nishibe, H. Yamadcra, Y. Nonomura, M. Takeuchi, J. Sakata, and Y., IEEE Trans. Magn. 32, 4965 (1996).

${ }^{5}$ A. L. Sukstanskii and V. Korenivski, J. Phys. D 34, 3337 (2001).

${ }^{6}$ F. Amalou and M. A. M. Gijs, Appl. Phys. Lett. 81, 1654 (2002).

${ }^{7}$ L.V. Panina, K. Mohri, K. Bushida, M. Noda, and T. Uchiyama, IEEE Trans. Magn. 31, 1249 (1995).

${ }^{8}$ D. P. Makhnovskiy, L. V. Panina, and D. J. Mapps, Phys. Rev. B 63, 144424 (2001).

${ }^{9}$ C. Dong, S. Chen, and T. Y. Hsu, J. Magn. Magn. Mater. 250, 288 (2002).

${ }^{10}$ C. Dong, S. Chen, and T. Y. Hsu, J. Magn. Magn. Mater. 263, 78(2003).

${ }^{11}$ A. Garcia-Arribas, J. M. Barandiaran, and D. de Cos, J. Magn. Magn. Mater. 320, e4 (2008).

${ }^{12}$ See http://www.comsol.com for comsol-multiphysics, Version 3.5a, COMSOL AB. 